\title{
Validation of a one year fracture prediction tool for absolute hip fracture risk in long term care residents
}

\author{
Ahmed M. Negm ${ }^{1,2^{*}}$ (D) George loannidis ${ }^{1,3}$, Micaela Jantzi ${ }^{4}$, Jenn Bucek ${ }^{4}$, Lora Giangregorio ${ }^{1,5}$, Laura Pickard ${ }^{1,3}$, \\ John P. Hirdes ${ }^{4}$, Jonathan D. Adachi ${ }^{3}$, Julie Richardson ${ }^{2,6}$, Lehana Thabane ${ }^{6}$ and Alexandra Papaioannou 13,6
}

\begin{abstract}
Background: Frail older adults living in long term care (LTC) homes have a high fracture risk, which can result in reduced quality of life, pain and death. The Fracture Risk Scale (FRS) was designed for fracture risk assessment in LTC, to optimize targeting of services in those at highest risk. This study aims to examine the construct validity and discriminative properties of the FRS in three Canadian provinces at 1-year follow up.

Methods: LTC residents were included if they were: 1) Adults admitted to LTC homes in Ontario (ON), British Columbia (BC) and Manitoba (MB) Canada; and 2) Received a Resident Assessment Instrument Minimum Data Set Version 2.0. After admission to LTC, one-year hip fracture risk was evaluated for all the included residents using the FRS (an eight-level risk scale, level 8 represents the highest fracture risk). Multiple logistic regressions were used to determine the differences in incident hip or all clinical fractures across the provinces and FRS risk levels. We examined the differences in incident hip or all clinical fracture for each FRS level across the three provinces (adjusted for age, BMI, gender, fallers and previous fractures). We used the C-statistic to assess the discriminative properties of the FRS for each province.

Results: Descriptive statistics on the LTC populations in ON $(n=29,848)$, BC $(n=3129)$, and MB $(n=2293)$ are: mean (SD) age 82 (10), 83 (10), and 84 (9), gender (female \%) 66, 64, and 70\% respectively. The incident hip fractures and all clinical fractures for FRS risk level were similar among the three provinces and ranged from 0.5 to $19.2 \%$ and 1 to $19.2 \%$ respectively. The overall discriminative properties of the FRS were similar between ON $(C$-statistic $=0.673)$, BC (C-statistic $=0.644)$ and MB (C-statistic $=0.649)$ samples.

Conclusion: FRS is a valid tool for identifying LTC residents at different risk levels for hip or all clinical fractures in three provinces. Having a fracture risk assessment tool that is tailored to the LTC context and embedded within the routine clinical assessment may have significant implications for policy, service delivery and care planning, and may improve care for LTC residents across Canada.
\end{abstract}

Keywords: Nursing home, Long term care, Hip fracture, Mortality, InterRAl prediction

\footnotetext{
* Correspondence: negmam@mcmaster.ca

${ }^{1}$ Geriatric Education and Research in Aging Sciences (GERAS), St Peter's

Hospital, 88 Maplewood Ave, Hamilton, ON, Canada

${ }^{2}$ School of Rehabilitation Sciences, IAHS 403, McMaster University, 1400 Main

St. W., Hamilton, Ontario L8S 1C7, Canada

Full list of author information is available at the end of the article
}

(c) The Author(s). 2018 Open Access This article is distributed under the terms of the Creative Commons Attribution 4.0 International License (http://creativecommons.org/licenses/by/4.0/), which permits unrestricted use, distribution, and reproduction in any medium, provided you give appropriate credit to the original author(s) and the source, provide a link to the Creative Commons license, and indicate if changes were made. The Creative Commons Public Domain Dedication waiver (http://creativecommons.org/publicdomain/zero/1.0/) applies to the data made available in this article, unless otherwise stated. 


\section{Background}

It has been estimated that 1 in 4 Canadians will be 65 years or older by 2036 [1]. As the population ages, a greater number of older adults will need residential support such as long-term care (LTC). LTC Residents are often frail, since their multiple physical and cognitive deficits place them at high risk of falls, disability, and death [2,3]. Hip fractures are the most common type of fracture in LTC (49\% of all fractures) [4]. They are more common in older adults living in LTC (49\%) than in the community $(29 \%)[4,5]$, and lead to more hospitalizations [6] and worsening health-related quality of life [7]. In Canada, $45 \%$ of LTC residents with hip fracture die within 12 months [8] and of the survivors, $48 \%$ are no longer ambulatory [8].

Hip fracture prediction and prevention in LTC residents receive little attention due to the multiple comorbidities and medical complexity of LTC residents $[9,10]$ and the challenges of predicting fracture in this population. It is difficult to identify LTC residents with high fracture risk, as the commonly used fracture risk assessment tools in Canada, including the Canadian Fracture Risk Assessment Tool (FRAX) and the Canadian Association of Radiologists and Osteoporosis Canada tool (CAROC) [11-14], are not valid or generalizable for residents of LTC $[15,16]$. FRAX and CAROC typically provide a 10-year fracture risk assessment timeframe, which is too long, given the mean 2.4-year life expectancy of LTC residents [17]. A recent study showed that FRAX (with bone mineral density) may predict incident hip fracture at one year [18]. Bone mineral density is heavily weighted in current fracture risk assessment protocols, but bone mineral density is not feasible to obtain in LTC. In addition, FRAX is not tailored to frail, institutionalized LTC residents. Thus, fracture prediction outputs of FRAX-Canada and CAROC may not be suitable for decision making and care planning among frail LTC residents [19, 20].

Recently, our team developed the Fracture Risk Scale (FRS) [21], a standardized outcome scale for identifying LTC residents at risk for fracture within one year. The FRS can be obtained from the Resident Assessment Instrument Minimum Data Set (RAI-MDS 2.0), which is a comprehensive, standardized assessment that is used upon admission and on a quarterly basis thereafter, to gather information on a wide range of socio-demographic and clinical characteristics [22-24]. The FRS was developed using Ontario residents' data from the RAI-MDS 2.0, the Discharge Abstract Database (DAD) and the National Ambulatory Care Reporting System (NACRS). However, the FRS was not externally validated (in a population other than the tool development sample), nor was its validity tested in other Canadian provinces. As a predictive model, the FRS' reproducibility (performing sufficiently accurate across new samples from the same target population) and transportability (performing well across samples from different but related source populations) [25] need to be tested prior widespread adoption. Therefore, we conducted this validity study to examine the FRS performance across a new sample and in different but related population of LTC residents in other provinces [21].

This study aims to: 1) assess the discriminative properties of the FRS using C-statistics and examine the construct validity of the FRS by comparing incident hip fractures and all clinical fractures (includes hip, spine, humerus, forearm, pelvis fractures) for each fracture risk levels in LTC residents across three Canadian provinces at 1-year follow up; 2) compare incident hip and all clinical fractures in LTC residents across three Canadian provinces; and 3) compare incident hip and all clinical fractures between the FRS risk levels. To examine the construct validity study of the FRS [26], we hypothesize that incident hip fractures for each fracture risk levels in LTC residents in the three Canadian provinces are not statistically different when type 1 error is $\leq 0.05$.

\section{Methods \\ Study design and population}

This retrospective cohort study uses data from the RAI-MDS 2.0. In Canada, LTC facilities provide living accommodation for people who need 24-h professional health services, personal care and services, including meals, laundry, housekeeping, frequent assistance with activities of daily living, on-site supervision or monitoring to ensure safety or well-being $[27,28]$. LTC residents were included if they: 1) were adults admitted to LTC homes in Canada from April 1st, 2006 to March 31st, 2010; and 2) were assessed with the RAI-MDS 2.0. LTC residents were excluded if they: 1 ) had multiple admissions; 2 ) reported on the RAI-MDS 2.0 to have end stage disease, were comatose, received hospice, or respite care; 3) were expected to have a short stay; 4) had the admission assessment completed more than 14 days after the date of admission (to exclude any assessment for existing residents); or 5) had no reassessments during the one-year follow-up. The project received ethics approval from the University of Waterloo Office of Research Ethics (ORE no 17045).

\section{Fracture rating scale}

The FRS is different from existing fracture risk assessment tools in that it does not use bone mineral density and includes fracture risk factors that are relevant to the long-term care (LTC) population [21]. Moreover, to ensure that it was valid for LTC residents and easily scalable, the FRS was designed and validated using large population-based datasets that include routinely collected data from LTC residents. The FRS was developed 
using decision tree analysis and the included items are walking in corridor (independent, supervision to extensive assistance or total assistance/walking did not occur), wandering (no wandering to infrequent wandering, less than daily wandering, or daily wandering), falling status within the past 30 days (no/yes), cognitive performance scale (intact cognition, borderline intact or mild impairment, or moderate to very severe impairment), transfer status (how resident moves between surfaces to and from; bed, chair, wheelchair, standing position), (independent to extensive assistance, or total assistance/transfer did not occur), age greater than 85 years (no/yes), body mass index (BMI) $(<18,18-30$, or $\left.>30 \mathrm{~kg} / \mathrm{m}^{2}\right)$, and previous fractures in past 180 days (no/yes) [21]. The FRS includes eight fracture risk level categories (level 1 represents the lowest and level 8 represents the highest fracture risk). Within the FRS, assessment of risk continues through the decision tree until a terminal risk level is identified. For example, individuals who can walk in corridor independently with BMI $>30$ are defined as fracture risk level 3. However, if BMI is between 18 and 30, risk level also depends on history of falls, fracture and cognitive performance.

\section{Incident fractures}

Over the course of one-year follow-up period, residents were classified as to the presence or absence of an incident fracture. To capture incident fractures, we accessed in-patient hospital records and emergency department records $[23,24]$. DAD is received directly from acute care facilities or their respective health/regional authority or ministry/department of health and it contains demographic, administrative and clinical data of all Canadian provinces except Quebec [29]. NACRS contains data for all hospital-based and community-based ambulatory care including day surgery, outpatient and community-based clinics and emergency departments [30]. NACRS data is received directly from participating facilities or from regional health authorities or ministries of health. Data collection methods may vary by facility. We were able to link DAD and NACRS data to RAI-MDS data for this analysis.

Incident fractures were defined using International Statistical Classification of Diseases and Related Health Problems, 10th Revision, Canada (ICD-10-CA) codes, captured in DAD and NACRS. The codes were selected using the Revised Framework for National Surveillance on Osteoporosis and Osteoporosis-related Fractures of the Public Health Agency of Canada [31]. A resident with one of the hip fracture codes (hip (S72.0, S72.1, S72.2) present on either a hospitalization or emergency department visit within one year after the admission assessment was code as having hip fracture. A resident with at least one of these codes within one year after the admission assessment was coded as having a fracture (hip (S72.0, S72.1, S72.2), spine (S22.0, S22.1, S32.0, S32.7, S32.8), humerus (S42.2), forearm (S52.x, S62.x) and pelvis (S32.1, S32.3, S32.4, S32.5, S32.7, S32.8)).

\section{Statistical analysis}

The study population demographics, prior falls and fractures, and fracture incidence estimates are expressed as mean (SD) for continuous data and counts and percentages for categorical data. Percent of incident hip fracture and all clinical fractures (hip, spine, humerus, forearm, pelvis fractures) for each FRS risk level in the three provinces were calculated. Multivariable logistic regression models were used to determine if incident hip fractures are different across the provinces and to calculate the odds ratio of incident hip fractures in each FRS risk level. In this logistic regression, the incident hip fractures were the dependent variable (DV) and provinces (Ontario was used as the reference group) and FRS risk levels (FRS risk level 1 was used as the reference level) were the independent variables (IV). We tested the significance of the interaction term of FRS and provinces in the logistic regression model to determine if the incident hip fracture in each FRS risk level is different across the provinces. The logistic regression analyses were adjusted for age, BMI, gender, fallers in the last 180 days, previous fracture, and the size of the residential home (small, medium or large). All the analyses were repeated using all incident fractures as DV. To assess the discriminative properties of the FRS for each province, we used the C-statistic. All the statistical analyses were conducted using SAS V.9.4 (SAS Institute).

\section{Results}

The final study sample includes 35,270 participants $(\mathrm{ON}=29,848, \mathrm{BC}=3129$ and $\mathrm{MB}=2293)$ and is displayed in Fig. 1. Of the eligible residents, $703(\mathrm{ON}=$ $447, \mathrm{BC}=169$ and $\mathrm{MB}=87$ ) were not included in the analysis due to data entry errors. Table 1 demonstrates the characteristics of LTC residents in the three provinces. Table 2 describes FRS items in LTC residents of the three provinces. Of the included residents, 3561 (10.1\%) residents died before the end of the one-year follow-up period. The characteristics of the excluded and included resident are comparable. Of the excluded residents $48.8 \%$ aged 85 years or older, and $67.9 \%$ are female. The age, gender distribution and comorbidities are similar across the provinces; with less than $1 \%$ of LTC residents having had a hospital admission within 3 months from LTC admission. Falls, incident fractures, and BMI were similar across the provinces as well. All of the Manitoba homes in the study were from one urban centre, as the remainder of the province does not use RAI-MDS.

Incident hip fractures and all clinical fractures in all risk levels ranged from 0.5 to $19.2 \%$ and 1 to $19.2 \%$ 


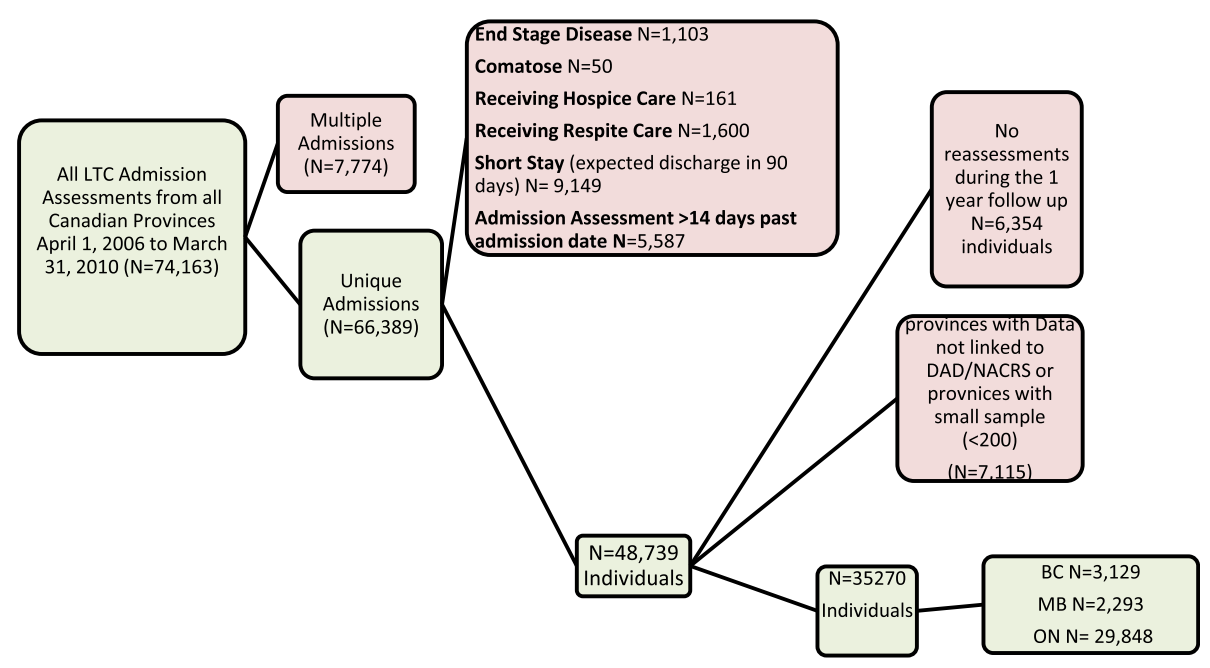

LTC: Long-term care, DAD: Discharge Abstract Database, NACRS: National Ambulatory Care Reporting System

Fig. 1 Study sample flow diagram

respectively (Figs. 2 and 3, respectively). Table 3 demonstrates the provincial fracture counts and percent by FRS levels. The incident hip fractures were comparable across the three provinces $(\mathrm{BC}=1.27(1.04,1.55), \mathrm{MB}=1.30$ $(1.04,1.62)$, when $\mathrm{ON}$ is the reference province). Also, there was no statistically significant difference in all clinical fractures $(\mathrm{BC}=0.97(0.81,1.16), \mathrm{MB}=0.84 \quad(0.68$, 1.04), when $\mathrm{ON}$ is the reference province) across the three provinces (Table 4). When adjusting for the provinces, the odds of incident hip fractures and all clinical fractures in all the FRS risk levels were significantly different compared to level 1 with consistently increasing odds of fractures with higher FRS risk levels, as shown in Table 4.

All the interaction terms associated with FRS risk levels and provinces were not statistically significant. This indicates the similarity of incident hip fractures and all clinical fractures for each FRS risk levels in the three provinces. The overall discriminative properties of the FRS were similar between $\mathrm{ON}(\mathrm{c}$-statistic $=0.67), \mathrm{BC}(\mathrm{c}$-statistic $=$ $0.64)$ and $M B(c$-statistic $=0.65)$ samples. The proportion of hip fractures that occurred in residents with a FRS level of 1-3 ranged from $4.9-7 \%$ across provinces, whereas the proportion of hip fractures that occurred in residents with a FRS of 6-8 was higher (>26\%).

\section{Discussion}

Our study demonstrates the validity of the FRS for predicting hip and all clinical fractures in LTC residents living in several Canadian provinces. As recommended by the Cosmin initiative [26], an international initiative that aims to improve the selection of health measurement instruments; hypothesis-testing construct validity is a critical component of evaluating outcome measures' psychometric properties. This study confirmed our hypothesis and showed that incident hip and all clinical fractures for each FRS risk levels in LTC residents in the three Canadian provinces were similar, confirming that the FRS is reproducible and transportable for LTC residents living in different geographical areas [25]. Therefore, our results confirmed that FRS can be used to identify LTC residents with high risk of hip or all fractures across different Canadian provinces.

We have previously shown that the FRS is able to identify LTC residents at highest risk of fracture in the initial FRS development study [21]. The FRS is an adequate reflection of the outcome of interest (one-year incident fracture). Our study builds on this work by examining FRS performance in LTC residents in three geographical areas. A clinical prediction tool performance is determined by assessing a model's calibration and discrimination [32]. Calibration is the agreement between prediction from the model (FRS model) and observed outcomes (incident hip and all clinical fractures) and indicates a model's predictive accuracy [33], which was shown in Table 4. Discrimination is the ability of the prediction model (FRS model) to set apart participants with and without the outcome of interest (incident hip and all clinical fractures); those with the outcome of interest should have a higher predicted risk compared to those who do not have it [33] which was also shown in Table 4.

Unlike commonly used fracture risk assessment tools (FRAX and CAROC), FRS is a unique one-year fracture prediction tool that is composed of risk factors specific to LTC residents. FRS is embedded in the RAI-MDS 2.0, which is completed within 14 days of a resident entering 
Table 1 Baseline characteristics of the study participants

\begin{tabular}{|c|c|c|c|}
\hline Variables & ON $(N=29,848)$ & $\mathrm{BC}(N=3129)$ & MB $(N=2293)$ \\
\hline Age, yrs. Mean (SD) & $82.1(9.6)$ & $83.0(9.6)$ & $83.5(9.3)$ \\
\hline Gender, Female, $n$ (\%) & $19,706(66.1)$ & $2023(64.7)$ & $2293(69.8)$ \\
\hline Married, $n(\%)$ & $8978(30.1)$ & $818(26.2)$ & $608(26.5)$ \\
\hline \multicolumn{4}{|l|}{ Chronic Disease Number, $n$ (\%) } \\
\hline Osteoporosis & $7247(24.3)$ & $663(21.2)$ & $353(15.4)$ \\
\hline Diabetes Mellitus & 7239 (24.3) & $610(19.5)$ & $472(20.6)$ \\
\hline Arthritis & $10,486(35.1)$ & $1058(33.8)$ & $811(35.4)$ \\
\hline Alzheimer's Disease & $5513(18.5)$ & $523(16.7)$ & $289(12.6)$ \\
\hline Cancer & $2935(9.8)$ & $280(9.0)$ & $279(12.2)$ \\
\hline Neuromuscular diseases & $47(0.2)$ & $11(0.4)$ & $1(0.04)$ \\
\hline Parkinson's Diseases & $81(0.3)$ & $2(0.1)$ & $6(0.3)$ \\
\hline Liver Disease & $69(0.2)$ & $7(0.2)$ & $7(0.3)$ \\
\hline Arteriosclerotic Diseases & $2004(6.7)$ & $199(6.4)$ & $127(5.5)$ \\
\hline \multicolumn{4}{|l|}{ Hospital admissions in past 90 days, $n(\%)$} \\
\hline No Visit & 29,603 (99.2) & $3106(99.3)$ & $2281(99.5)$ \\
\hline$\geq 1$ Visit & $245(0.8)$ & $23(0.7)$ & $12(0.5)$ \\
\hline \multicolumn{4}{|l|}{ Emergency room visits in past 90 day, $\mathrm{n} n(\%)$} \\
\hline No Visit & $26,590(89.1)$ & $2927(93.5)$ & $2232(97.3)$ \\
\hline$\geq 1$ Visit & $3258(10.9)$ & $202(6.5)$ & $61(2.7)$ \\
\hline Number of prescribed medications, Mean (SD) & $9.7(4.63)$ & $8.45(4.04)$ & $8.02(4.66)$ \\
\hline \multicolumn{4}{|c|}{ Changes in Health, End-Stage Disease, Signs, and Symptoms (CHESS Score), $n$ (\%) } \\
\hline 0 & $110,18(58.69)$ & $1309(68.64)$ & $664(72.49)$ \\
\hline 1 & $4665(24.85)$ & $362(18.98)$ & $188(20.52)$ \\
\hline 2 & $2179(11.61)$ & $176(9.23)$ & $51(5.57)$ \\
\hline 3 & $692(3.69)$ & $49(2.57)$ & $11(1.20)$ \\
\hline 4 & $219(1.17)$ & $11(0.58)$ & $2(0.22)$ \\
\hline 5 & 0 & 0 & 0 \\
\hline \multicolumn{4}{|l|}{ Home size, $n(\%)$} \\
\hline Small ( $\leq 50$ beds) & $583(1.95)$ & $276(8.82)$ & 0 \\
\hline Med (51-99 beds) & $511,9(17.15)$ & $103,7(33.14)$ & $278(12.12)$ \\
\hline Large $(\geq 100$ beds & $24,146(80.90)$ & $181,6(58.04)$ & $2015(87.88)$ \\
\hline Overall case mix index of the residents at Baseline, Mean (SD) & $0.64(0.18)$ & $0.57(0.16)$ & $0.58(0.14)$ \\
\hline \multicolumn{4}{|l|}{ Ownership, $n$ (\%) } \\
\hline Public/religious/not for profit & $127,41(42.69)$ & $157,3(50.27)$ & $122,3(53.34)$ \\
\hline Private & 171,07 (57.31) & $155,6(49.73)$ & $107,0(46.66)$ \\
\hline \multicolumn{4}{|l|}{ Rurality, n (\%) } \\
\hline Urban & $255,96(85.75)$ & $272,3(87.02)$ & 2293 (100) \\
\hline Rural & $410,5(13.75)$ & 401 (12.82) & 0 \\
\hline
\end{tabular}

a home and quarterly thereafter. In addition, the FRS can be obtained from the interRAI Long Term Care Facility assessment, which is the successor to the RAI-MDS 2.0 [24, 34]. Thus, FRS can be easily and regularly implemented in LTC without burden on the LTC staff $[9,10,35,36]$. As we demonstrated the FRS' validity, we suggest it can be used for LTC resident care planning across Canada and possibly internationally. Recently, Fracture Risk Assessment in Long-term Care (FRAiL) tool has been developed, which aims to predict two-year hip fracture risk in nursing home residents [16]. The FRAiL tool developed using RAI-MDS 2.0 assessment 
Table 2 Fracture rating scale items in the three provinces

\begin{tabular}{|c|c|c|c|}
\hline Fracture rating scale items & ON $(N=29,848)$ & $B C(N=3129)$ & MB $(N=2293)$ \\
\hline \multicolumn{4}{|l|}{ Age group } \\
\hline 18 to 64 & $1700(5.7)$ & $162(5.2)$ & $92(4.0)$ \\
\hline 65 to 74 & $3128(10.5)$ & $325(10.4)$ & $198(8.6)$ \\
\hline 75 to 84 & $11,300(37.9)$ & $1028(32.9)$ & $799(34.9)$ \\
\hline $85+$ & $13,708(45.9)$ & $1613(51.6)$ & $1202(52.5)$ \\
\hline \multicolumn{4}{|l|}{ Body Mass Index } \\
\hline$<18$ & $2396(8.0)$ & $423(13.5)$ & $254(11.1)$ \\
\hline $18-29$ & $22,252(74.6)$ & $2310(73.8)$ & $1677(73.1)$ \\
\hline $30+$ & $4753(15.9)$ & $396(12.7)$ & $362(15.8)$ \\
\hline \multicolumn{4}{|l|}{ Wandering Frequency } \\
\hline Not in last 7 days & $22,825(76.5)$ & $2340(74.8)$ & $1856(80.9)$ \\
\hline 1 to 3 days (in past 7 days) & $1925(6.5)$ & $275(8.8)$ & $141(6.2)$ \\
\hline 4 to 6 days (in past 7 days) & $1614(5.4)$ & $197(6.3)$ & $83(3.6)$ \\
\hline Daily (in past 7 days) & $3484(11.7)$ & $317(10.1)$ & $213(9.3)$ \\
\hline \multicolumn{4}{|l|}{ Walking in corridor } \\
\hline Independent & $10,530(35.3)$ & $1500(47.9)$ & $1016(44.3)$ \\
\hline Supervision & $4477(15.0)$ & $294(9.4)$ & $223(9.7)$ \\
\hline Limited assistance & $2789(9.3)$ & $223(7.1)$ & $197(8.59)$ \\
\hline Extensive assistance & $2086(7.0)$ & $107(3.4)$ & $74(3.2)$ \\
\hline Total dependence & $381(1.3)$ & $27(0.9)$ & $35(1.5)$ \\
\hline Activity did not occur & $9585(32.1)$ & $978(31.3)$ & 748 (32.6) \\
\hline \multicolumn{4}{|l|}{ Transfer status } \\
\hline Independent & $9569(32.1)$ & $1510(48.3)$ & $1054(46.0)$ \\
\hline Supervision & $3576(12.0)$ & $254(8.1)$ & $190(8.29)$ \\
\hline Limited assistance & $4662(15.6)$ & $468(15.0)$ & $411(17.9)$ \\
\hline Extensive assistance & $7140(23.9)$ & $481(15.4)$ & $255(11.1)$ \\
\hline Total dependence & $4806(16.1)$ & $396(12.7)$ & $382(16.7)$ \\
\hline Activity did not occur & $95(0.3)$ & $20(0.6)$ & $1(0.04)$ \\
\hline \multicolumn{4}{|l|}{ Cognitive performance scale } \\
\hline Intact & $5159(17.3)$ & $400(12.8)$ & $258(11.3)$ \\
\hline Borderline Intact & $4517(15.1)$ & $478(15.3)$ & $370(16.1)$ \\
\hline Mild & $6270(21.0)$ & $657(21.0)$ & $440(19.2)$ \\
\hline Moderate to very severe & $8697(29.1)$ & $1037(33.1)$ & $802(35.0)$ \\
\hline Moderate Severe & $1650(5.5)$ & $143(4.6)$ & $104(4.5)$ \\
\hline Severe & $2538(8.5)$ & $354(11.3)$ & $256(11.2)$ \\
\hline Very Severe & $1017(3.4)$ & $60(1.9)$ & $63(2.8)$ \\
\hline \multicolumn{4}{|l|}{ Falls within the last 30 days, $n$ (\%) } \\
\hline No Falls & $24,620(82.5)$ & $2569(82.1)$ & $1923(83.9)$ \\
\hline$\geq 1$ Fall & $5228(17.5)$ & $560(17.9)$ & $370(16.1)$ \\
\hline Prior hip fracture in last 180 days, $n$ (\%) & $179,088(6)$ & $18,774(6)$ & 6879 (3) \\
\hline
\end{tabular}

of nursing home residents in the United States. FRAiL tools included Fifteen items to predict hip fracture: older age, white race, female, impaired cognition, activities of daily living independence, locomotion independence, urinary continence, previous falls, transfer independence, easily distracted, wandering, absence of osteoarthritis, absence of pressure ulcer, low BMI, and diabetes. There are some common items in FRS and FRAiL tools such as 


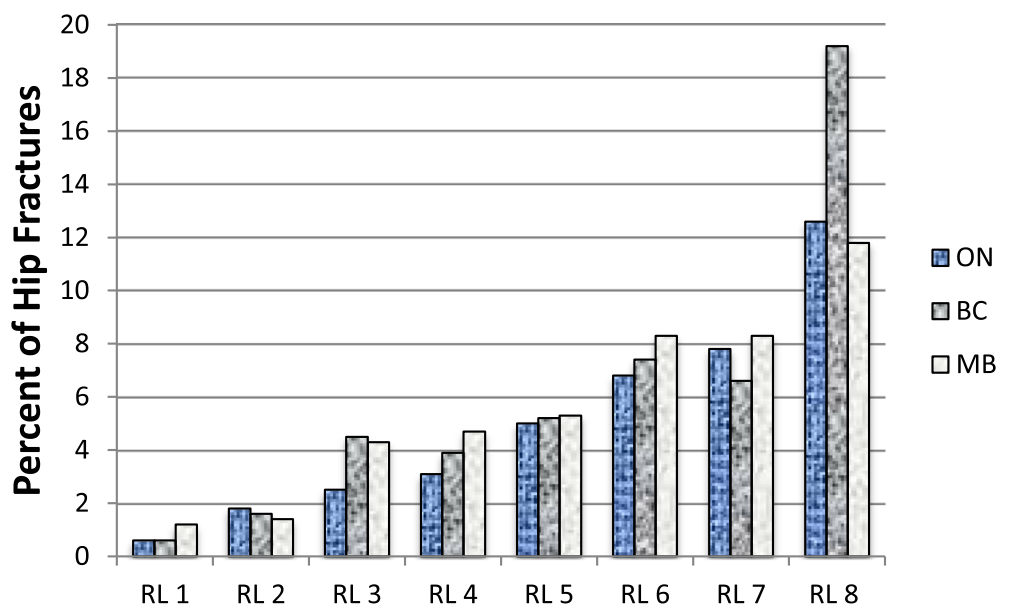

Fig. 2 Incident Hip fracture for Fracture Rating Scale risk levels

wandering, falling, cognitive performance scale, transfer status, age, BMI, and previous fractures.

Recommendations for preventing fracture in LTC have been developed to provide non-pharmacologic and pharmacologic strategies for fracture prevention in frail older adults living in LTC [37]. However, there is a current gap in osteoporosis treatment and fracture prevention in LTC residents [38-43]. One of the barriers to implement fracture prevention guideline is the lack of information about fracture risk assessment [44]. The easily implemented and validated FRS tool may overcome this barrier. To help LTC clinicians prescribing the appropriate intervention to residents who are identified as "at risk", our team will develop and implement an electronic Clinical Assessment Protocol (CAP) in LTCs. The Fracture Risk CAP will automatically produce recommendations for residents based on their FRS fracture risk level and will inform clinical decision making as part of the person-centered care planning process to fill the gap of fracture prevention in LTC. Other CAPs have been developed to draw the attention of the healthcare provider to a matter (such as Activities of daily living, delirium and Cardiorespiratory) that can be improved and should be considered in LTC residents' care plan [45].

Strengths of this study include the use of a large, representative sample of LTC residents with RAI-MDS 2.0 data linked with DAD and NACRS. As it is recommended to externally validate the model in samples from related source populations (LTC residents in other provinces) [25], we examined the performance of the FRS outside of the development sample. Assessing the relation between the development and validation samples allowed us to explain the FRS (clinical) transportability and (statistical) reproducibility [25].

We acknowledge that our study has limitations. We excluded LTC residents with short life expectancy based

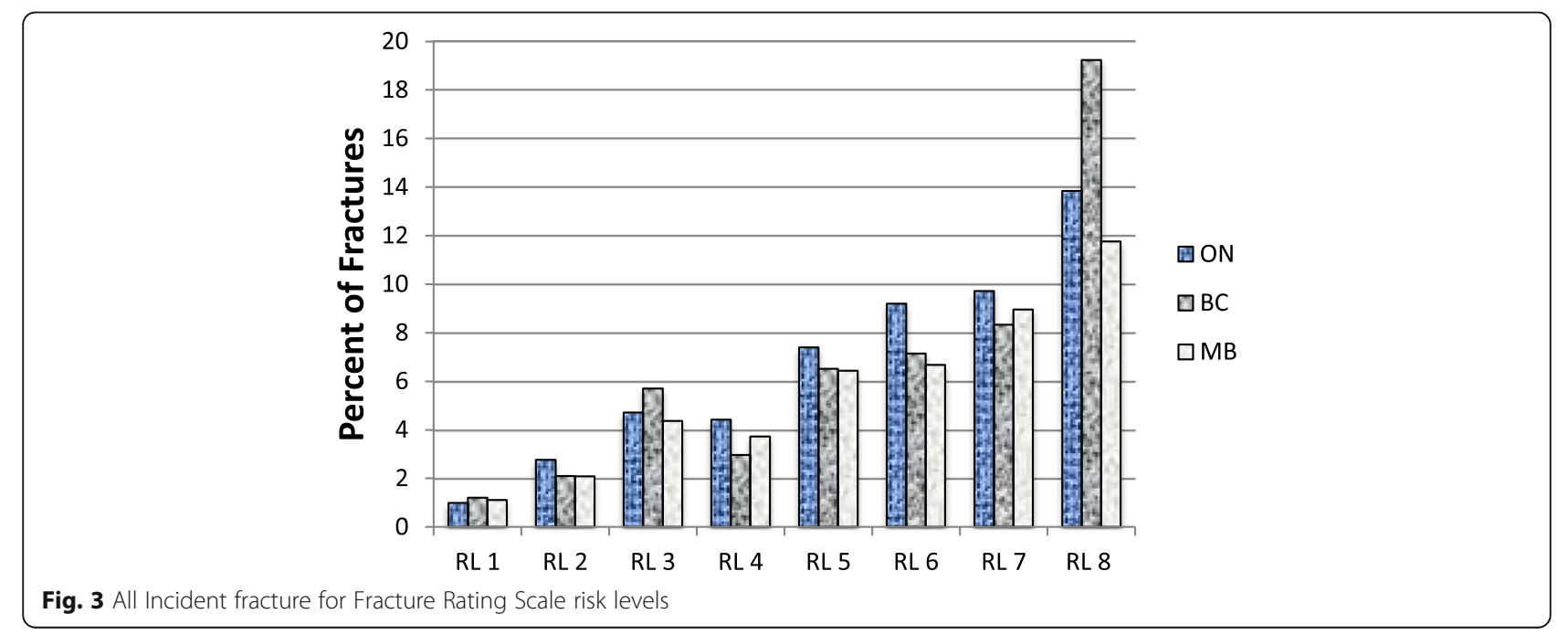


Table 3 Provincial fracture counts and percent by FRS levels

\begin{tabular}{|c|c|c|c|c|c|c|}
\hline \multirow[b]{2}{*}{ FRS risk levels } & \multicolumn{3}{|c|}{ Hip Fracture $n(\%)$} & \multicolumn{3}{|c|}{ All Fractures n (\%) } \\
\hline & $\mathrm{ON}$ & $B C$ & MB & $\mathrm{ON}$ & BC & MB \\
\hline 1 & $15(0.5)$ & $2(0.8)$ & $3(1.1)$ & $30(1.0)$ & $3(1.2)$ & $3(1.1)$ \\
\hline 2 & $53(1.8)$ & $5(1.5)$ & $4(1.7)$ & $82(2.8)$ & $7(2.1)$ & $5(2.1)$ \\
\hline 3 & $194(2.6)$ & $39(4.4)$ & $29(4.2)$ & $353(4.7)$ & $51(5.7)$ & $30(4.4)$ \\
\hline 4 & $174(2.5)$ & $13(2.4)$ & $14(3.7)$ & $305(4.4)$ & $16(3.0)$ & $14(3.7)$ \\
\hline 5 & $238(4.6)$ & $31(5.2)$ & $21(5.4)$ & $380(7.4)$ & $39(6.5)$ & $25(6.4)$ \\
\hline 6 & $47(6.8)$ & $2(7.1)$ & $1(6.7)$ & $64(9.2)$ & $2(7.1)$ & $1(6.7)$ \\
\hline 7 & $205(6.7)$ & $20(6.9)$ & $17(8.0)$ & $296(9.7)$ & $24(8.3)$ & $19(9.0)$ \\
\hline 8 & $20(12.6)$ & $5(19.2)$ & $2(11.8)$ & $22(13.8)$ & $5(19.2)$ & $2(11.8)$ \\
\hline Total $^{a}$ & 959 (3.2) & $124(4.0)$ & $92(4.0)$ & $1553(5.2)$ & $154(4.9)$ & $100(4.4)$ \\
\hline
\end{tabular}

${ }^{a}=$ Total fractures do not add up due to excluding participants with data entry error

on the data from the RAI-MDS 2.0 (participants who have end stage disease, were comatose, received hospice, or respite care, or expected a short stay), as we aim to inform decisions related to fracture prediction and prevention for those whom life expectancy is 1 year or greater. Therefore, our findings may not be generalizable to some LTC residents. We included participants who died before the end of the one-year follow up in the analysis, which may underestimate the incident fracture, as these people may have a higher likelihood of fracture. Competing risk framework was not employed in the analyses, as we excluded individuals who were likely to die within a year apriori. Despite not employing competing risk framework, our result shows that FRS performs reasonably well in identifying at risk people. We

Table 4 Differences in Hip Fracture across provinces and Fracture risk scale risk levels

\begin{tabular}{lll}
\hline Variables & $\begin{array}{ll}\text { Hip Fractures } \\
\text { OR }(\mathrm{Cl})\end{array}$ & $\begin{array}{l}\text { All Fractures } \\
\mathrm{OR}(\mathrm{Cl})\end{array}$ \\
\hline Provinces & \\
ON & 1 & 1 \\
$\mathrm{BC}$ & $1.27(1.04,1.55)$ & $0.97(0.81,1.16)$ \\
$\mathrm{MB}$ & $1.30(1.04,1.62)$ & $0.84(0.68,1.04)$
\end{tabular}

FRS risk levels

\begin{tabular}{lll}
1 & 1 & 1 \\
2 & $3.08(1.86,5.11)$ & $2.64(1.79,3.89)$ \\
3 & $5.56(3.52,8.80)$ & $5.28(3.74,7.45)$ \\
4 & $4.29(2.68,6.92)$ & $3.84(2.68,5.50)$ \\
5 & $8.02(5.06,12.72)$ & $6.93(4.90,9.81)$ \\
6 & $12.17(7.15,20.73)$ & $8.97(5.90,13.65)$ \\
7 & $10.52(6.60,16.79)$ & $8.36(5.86,11.91)$ \\
8 & $17.00(10.15,35.55)$ & $10.90(6.41,18.59)$ \\
\hline
\end{tabular}

OR Odds Ratio, Cl 95\% Confidence Interval, ON Ontario, $B C$ British Colombia, MB Manitoba, FRS Fracture Rating Scale. The analyses were adjusted for age, $\mathrm{BMl}$, gender, fallers in the last 180 days, previous fracture, and Home size (small, medium or large) used a validated framework to capture incident fractures (Revised Framework for National Surveillance on Osteoporosis and Osteoporosis-related Fractures of the Public Health Agency of Canada [31]), however, vertebral and other fractures are likely not fully captured. Our study was limited to the variables collected in the RAI-MDS 2.0 and may not have captured all relevant risk factors for hip or all clinical fractures in LTC residents.

\section{Conclusion}

The FRS is a valid tool for identifying LTC residents at different risk levels for hip or all clinical fractures in three provinces. Having a fracture risk assessment tool that is tailored to the LTC context and embedded within the routine clinical assessment may have significant implications for policy, service delivery and care planning, and may improve care for LTC residents across Canada.

\section{Abbreviations}

BC: British Columbia; CAP: Clinical Assessment Protocol; CAROC: Canadian Association of Radiologists and Osteoporosis Canada tool; Cl: Confidence Interval; DAD: Canadian Discharge Abstract Database; DV: Dependent Variable; FRAX: Fracture Risk Assessment Tool; FRS: Fracture Rating Scale; ICD10 code: International Classification of Disease-10 code; IV: Independent Variable; LTC: Long Term Care; MB: Manitoba; NACRS: National Ambulatory Care Reporting System; ON: Ontario; RAI-MDS 2.0: Resident Assessment Instrument Minimum Data Set: SD: Standard Deviation

\section{Acknowledgments}

Not applicable

\section{Funding}

The Ministry of Health and Long-Term Care through the Ontario Osteoporosis Strategy for Long-Term Care. AN received PhD studentship funding from Osteoporosis Canada to complete this project. In addition, funding for the analytic dataset was provided by the public Health Agency of Canada through a grant to JPH (Project\#6271-15-2010/3970773). The funding sources had no role in the design and conduct of the study; collection, management, analysis and interpretation of the data; or preparation, review or approval of the manuscript

Availability of data and materials

The data that support the findings of this study will not be shared. Our access to the de-identified record-level data is provided through the Canadian Institute for Health Information $(\mathrm{CIHI})$, under license from interRAI, the 
international non-profit organization that holds the copyright for the assessment itself. Other interested researchers could also submit requests to $\mathrm{ClHI}$ if they wish to access the study data.

\section{Authors' contributions}

AN was responsible for study design, data analysis and interpretation and drafting of manuscript, $\mathrm{MJ}$ and $\mathrm{Gl}$ was responsible for study design, data analysis and interpretation and drafting of manuscript. JB, JDA, LG, LT, JR, $\mathrm{JH}, \mathrm{LP}$ and AP were responsible for study design, data interpretation and critical review of the manuscript. All authors read and approved the final manuscript.

\section{Ethics approval and consent to participate}

The project received ethics approval from the University of Waterloo Office of Research Ethics (ORE no 17045). The need for consent is deemed unnecessary because the MDS 2.0 assessments are performed in Long-term care homes on all residents as part of regular clinical practice that is mandated by the Ministries of Health in the relevant Canadian provinces. For example, in Ontario, the Health Care Consent Act provides rules with respect to consent to treatment in a Health Care environment. It specifically states that the "assessment or examination of a person to determine the general nature of the person's condition is not considered to be treatment and would therefore not require consent".

\section{Consent for publication}

Not applicable.

\section{Competing interests}

All authors declare no support from any organisation for the submitted work; no financial relationships with any organisations that might have an interest in the submitted work in the previous three years; no other relationships or activities that could appear to have influenced the submitted work (GI, MJ, JB, JPH, LT, JR). JPH reported funding from PHAC. AN reported funding from Osteoporosis Canada. LG reports personal fees from ICON. AP and LP report grants from Ontario Ministry of Health and Long-Term Care Ontario Osteoporosis Strategy. JDA reports grants and personal fees from Amgen, grants and personal fees from Eli Lilly, personal fees from AgNovos, during the conduct of the study; non-financial support from Osteoporosis Canada, non-financial support from International Osteoporosis Foundation, outside the submitted work.

\section{Publisher's Note}

Springer Nature remains neutral with regard to jurisdictional claims in published maps and institutional affiliations.

\section{Author details}

'Geriatric Education and Research in Aging Sciences (GERAS), St Peter's Hospital, 88 Maplewood Ave, Hamilton, ON, Canada. ${ }^{2}$ School of Rehabilitation Sciences, IAHS 403, McMaster University, 1400 Main St. W. Hamilton, Ontario L8S 1C7, Canada. ${ }^{3}$ Department of Medicine, McMaster University, Hamilton, Ontario, Canada. ${ }^{4}$ School of Public Health and Health Systems, University of Waterloo, Waterloo, Ontario, Canada. ${ }^{5}$ Department of Kinesiology and Schlegel-UW Research Institute for Aging, University of Waterloo, Waterloo, Ontario, Canada. ${ }^{6}$ Department of Health Research Methods, Evidence, and Impact, McMaster University, 1280 Main St West, Hamilton, ON, Canada.

Received: 18 July 2018 Accepted: 11 December 2018 Published online: 27 December 2018

\section{References}

1. Canada S. Living arrangements of seniors. Census in brief No. 4.; 2011.

2. Abellan van Kan G, Rolland Y, Houles M, Gillette-Guyonnet S, Soto M, Vellas B. The assessment of frailty in older adults. Clin Geriatr Med. 2010;26(2):275-86

3. Bergman H, Ferrucci L, Guralnik J, Hogan DB, Hummel S, Karunananthan S, et al. Frailty: an emerging research and clinical paradigm--issues and controversies. J Gerontol A Biol Sci Med Sci. 2007;62(7):731-7.

4. Papaioannou A, Kennedy CC, loannidis G, Cameron C, Croxford R, Adachi $J \mathrm{D}$, et al. Comparative trends in incident fracture rates for all long-term care and community-dwelling seniors in Ontario, Canada, 2002-2012. Osteoporos Int. 2016;27(3):887-97.
5. Tarride JE, Burke N, Leslie WD, Morin SN, Adachi JD, Papaioannou A, et al. Loss of health related quality of life following low-trauma fractures in the elderly. BMC Geriatr. 2016;16:84.

6. Ronald LA, McGregor MJ, McGrail KM, Tate RB, Broemling AM Hospitalization rates of nursing home residents and community-dwelling seniors in British Columbia. Can J Aging. 2008;27(1):109-15.

7. Dyer SM, Crotty M, Fairhall N, Magaziner J, Beaupre LA, Cameron ID, et al. A critical review of the long-term disability outcomes following hip fracture. BMC Geriatr. 2016;16:158.

8. Beaupre LA, Jones CA, Johnston DW, Wilson DM, Majumdar SR. Recovery of function following a hip fracture in geriatric ambulatory persons living in nursing homes: prospective cohort study. J Am Geriatr Soc. 2012;60(7):1268-73.

9. Doupe M, St John P, Chateau D, Strang D, Smele S, Bozat-Emre S, et al. Profiling the multidimensional needs of new nursing home residents: evidence to support planning. J Am Med Dir Assoc. 2012;13(5):487 e9-17.

10. A B. An Overview of Long-Term Care in Canada and Selected Provinces and Territories.; 2007.

11. Siminoski K, Leslie WD, Frame H, Hodsman A, Josse RG, Khan A, et al. Recommendations for bone mineral density reporting in Canada: a shift to absolute fracture risk assessment. J Clin Densitom. 2007;10(2):120-3.

12. Siminoski $K$, Leslie WD, Frame $H$, Hodsman A, Josse RG, Khan A, et al. Recommendations for bone mineral density reporting in Canada. Can Assoc Radiol J. 2005;56(3):178-88.

13. Kanis JA, Johnell $O$, De Laet $C$, Johansson $H$, Oden A, Delmas $P$, et al. A meta-analysis of previous fracture and subsequent fracture risk. Bone. 2004; 35(2):375-82.

14. Leslie WD, Lix LM, Langsetmo L, Berger C, Goltzman D, Hanley DA, et al. Construction of a $\operatorname{FRAX}(\mathrm{R})$ model for the assessment of fracture probability in Canada and implications for treatment. Osteoporos Int. 2011;22(3):817-27.

15. Bravo G, Dubois MF, De Wals P, Hebert R, Messier L. Relationship between regulatory status, quality of care, and three-year mortality in Canadian residential care facilities: a longitudinal study. Health Serv Res. 2002;37(5): 1181-96.

16. Living arrangements of seniors. Catalogue no. 98-312-X2011003: Statistics Canada; 2011.

17. Jones AL, Dwyer LL, Bercovitz AR, Strahan GW. The National Nursing Home Survey: 2004 overview. Vital Health Stat 13. 2009(167):1-155.

18. Leslie WD, Majumdar SR, Morin SN, Lix LM, Johansson H, Oden A, et al. FRAX for fracture prediction shorter and longer than 10 years: the Manitoba BMD registry. Osteoporos Int. 2017;28(9):2557-64.

19. Cox L, Kloseck M, Crilly R, McWilliam C, Diachun L. Underrepresentation of individuals 80 years of age and older in chronic disease clinical practice guidelines. Can Fam Physician. 2011;57(7):e263-9.

20. Mutasingwa DR, Ge H, Upshur RE. How applicable are clinical practice guidelines to elderly patients with comorbidities? Can Fam Physician. 2011; 57(7):e253-62.

21. Ioannidis G, Jantzi M, Bucek J, Adachi JD, Giangregorio L, Hirdes J, et al. Development and validation of the fracture risk scale (FRS) that predicts fracture over a 1-year time period in institutionalised frail older people living in Canada: an electronic record-linked longitudinal cohort study. BMJ Open. 2017;7(9):e016477.

22. Morris JN, Nonemaker S, Murphy K, Hawes C, Fries BE, Mor V, et al. A commitment to change: revision of HCFA's RAI. J Am Geriatr Soc. 1997; 45(8):1011-6.

23. Hawes C, Morris JN, Phillips CD, Fries BE, Murphy K, Mor V. Development of the nursing home resident assessment instrument in the USA. Age Ageing. 1997;26(Suppl 2):19-25.

24. Hirdes JP, Ljunggren G, Morris JN, Frijters DH, Finne Soveri H, Gray L, et al. Reliability of the interRAI suite of assessment instruments: a 12-country study of an integrated health information system. BMC Health Serv Res. 2008:8:277.

25. Debray TP, Vergouwe Y, Koffijberg H, Nieboer D, Steyerberg EW, Moons KG. A new framework to enhance the interpretation of external validation studies of clinical prediction models. J Clin Epidemiol. 2015;68(3):279-89.

26. Mokkink LB, Terwee CB, Patrick DL, Alonso J, Stratford PW, Knol DL, et al. The COSMIN study reached international consensus on taxonomy, terminology, and definitions of measurement properties for health-related patient-reported outcomes. J Clin Epidemiol. 2010;63(7):737-45.

27. Hirdes JP, Mitchell L, Maxwell CJ, White N. Beyond the 'iron lungs of gerontology': using evidence to shape the future of nursing homes in Canada. Can J Aging. 2011;30(3):371-90. 
28. Long-term facilities-based care 2004 [Available from: https://www.canada. ca/en/health-canada/services/home-continuing-care/long-term-facilitiesbased-care.html.

29. Discharge Abstract Database Metadata (DAD): Canadian Institute of Health Information; 2018 [Available from: https://www.cihi.ca/en/dischargeabstract-database-metadata.

30. National Ambulatory Care Reporting System Metadata (NACRS): Canadian Institute of Health Information; 2018 [Available from: https://www.cihi.ca/en/ national-ambulatory-care-reporting-system-metadata.

31. O'Donnell S, Canadian Chronic Disease Surveillance System Osteoporosis Working G. Use of administrative data for national surveillance of osteoporosis and related fractures in Canada: results from a feasibility study. Archives of osteoporosis. 2013;8:143-.

32. Steyerberg EW, Vickers AJ, Cook NR, Gerds T, Gonen M, Obuchowski N, et al. Assessing the performance of prediction models: a framework for traditional and novel measures. Epidemiology. 2010;21(1):128-38.

33. EW S. Clinical prediction models: a practical approach to development, validation, and updating. New York: Springer; 2009.

34. Onder G, Carpenter I, Finne-Soveri H, Gindin J, Frijters D, Henrard JC, et al. Assessment of nursing home residents in Europe: the services and health for elderly in long TERm care (SHELTER) study. BMC Health Serv Res. 2012;12:5.

35. Chamberlain SA, Gruneir A, Hoben M, Squires JE, Cummings GG, Estabrooks CA. Influence of organizational context on nursing home staff burnout: a cross-sectional survey of care aides in Western Canada. Int J Nurs Stud. 2017;71:60-9.

36. Bowers BJ, Esmond S, Jacobson N. The relationship between staffing and quality in long-term care facilities: exploring the views of nurse aides. J Nurs Care Qual. 2000;14(4):55-64 quiz 73-5.

37. Papaioannou A, Santesso N, Morin SN, Feldman S, Adachi JD, Crilly R, et al. Recommendations for preventing fracture in long-term care. CMAJ. 2015; 187(15):1135-44 E450-61.

38. Wall M, Lohfeld L, Giangregorio L, loannidis G, Kennedy CC, Moser A, et al. Fracture risk assessment in long-term care: a survey of long-term care physicians. BMC Geriatr. 2013;13:109.

39. Kennedy CC, loannidis G, Thabane L, Adachi JD, O'Donnell D, Giangregorio $L M$, et al. Osteoporosis prescribing in long-term care: impact of a provincial knowledge translation strategy. Can J Aging. 2015;34(2):137-48.

40. Jachna CM, Shireman TI, Whittle J, Ellerbeck EF, Rigler SK. Differing patterns of antiresorptive pharmacotherapy in nursing facility residents and community dwellers. J Am Geriatr Soc. 2005;53(8):1275-81.

41. Kennedy CC, loannidis G, Thabane L, Adachi JD, Marr S, Giangregorio LM, et al. Successful knowledge translation intervention in long-term care: final results from the vitamin $\mathrm{D}$ and osteoporosis study (ViDOS) pilot cluster randomized controlled trial. Trials. 2015;16:214.

42. Colon-Emeric CS, Lyles KW, House P, Levine DA, Schenck AP, Allison J, et al. Randomized trial to improve fracture prevention in nursing home residents. Am J Med. 2007:120(10):886-92.

43. Giangregorio LM, Jantzi M, Papaioannou A, Hirdes J, Maxwell CJ, Poss JW. Osteoporosis management among residents living in long-term care. Osteoporos Int. 2009;20(9):1471-8

44. Alamri SH, Kennedy CC, Marr S, Lohfeld L, Skidmore CJ, Papaioannou A. Strategies to overcome barriers to implementing osteoporosis and fracture prevention guidelines in long-term care: a qualitative analysis of action plans suggested by front line staff in Ontario. Canada BMC Geriatr. 2015;15:94.

45. OECD, Union E. A Good Life in Old Age?2013.

Ready to submit your research? Choose BMC and benefit from:

- fast, convenient online submission

- thorough peer review by experienced researchers in your field

- rapid publication on acceptance

- support for research data, including large and complex data types

- gold Open Access which fosters wider collaboration and increased citations

- maximum visibility for your research: over $100 \mathrm{M}$ website views per year

At $\mathrm{BMC}$, research is always in progress.

Learn more biomedcentral.com/submissions 